\title{
TWO-DIMENSIONAL SOURCE POTENTIALS IN A TWO-FLUID MEDIUM FOR THE MODIFIED HELMHOLTZ'S EQUATION
}

\author{
B. N. MANDAL and R. N. CHAKRABARTI \\ Department of Applied Mathematics \\ University College of Science \\ 92, A. P. C. Road \\ Calcutta - 700009 , India \\ (Received March 10, 1985)
}

ABSTRACT. Velocity potentials describing the irrotational infinitesimal motion of two superposed inviscid and incompressible fluids under gravity with a horizontal plane of mean surface of separation, are derived due to a vertical line source present in either of the fluids, whose strength, besides being harmonic in time, varies sinusiodally along its length. The technique of deriving the potentials here is an extension of the technique used for the case of only time harmonic vertical line source. The present case is concerned with the two-dimensional modified Helmholtz's equation while the previous is concerned with the two-dimensional Laplace's equation.

KEY WORDS AND PHRASES. Modified Helmholtz's equation, two-fluid medium, source potentials, surface of separation.

1980 AMS SUBJECT CLASSIFICATION CODES: 76B15, 31A05, 35A45.

\section{INTRODUCTION.}

Velocity potentials due to the presence of different types of singularities in an incompressible and inviscid one-fluid medium, assuming irrotational motion of small amplitudes, play an important role in dealing with problems involving radiation or scattering of surface waves by obstacles present in the medium. These problems can be reduced to equivalent problems of solving some singular integral equations of second kind in general, by a suitable use of Green's integral theorem in the fluid medium with the help of these singular potentials (generally called Green's function). Thorne [1] gave a survey of the fundamental singularities submerged in an one-fluid medium and Rhodes-Robinson [2] modified it to include the effect of surface tension at the free surface (FS). Gorgui and Kassem [3] considered a two-fluid medium and obtained potentials due to oscillating line and point singularities submerged in either of the fluids. The upper fluid of the two-fluid medium considered in [3] is extended infinitely upwards and the lower fluid is of either infinite or finite depth below the mean surface of separation (SS). Later the model is modified to include a number of generalizations, e.g. presence of interfacial surface tension in the SS (cf. Rhodes-Robinson [4], Mandal [5]) upper fluid of finite depth with a free surface with or without surface tension (cf. Chakrabarti and Mandal [6], Chakrabarti [7], Kassem [8]).

In problems dealing with the scattering of obliquely incident surface waves in an ine-fluid medium by horizontal plane barriers (cf. Heins [9], Green and Heins [10] 
etc.) or vertical plane barriers (cf. Mandal and Goswami [11], [12], [13]), halfimmersed or fully submerged infinitely long circular cylinder (cf. Mandal and Goswami [14], Levine [15], by exploiting the geometry of the obstacles, the velocity potential can be assumed to have a harmonic variation in the lateral (z) direction, same as the incident wave field. Thus the potential function satisfies a two-dimensional reduced Helnholtz's equation. Hence the problems are essentially boundary value problems (BVP) involving the Helmholtz's equation, and the construction of a two-dimensional source potential (the Green's function) is necessary to reduce the BVP's to equivalent integral equations. Both for infinite and finite constant depth of fluid, this source potential can be constructed by the method of Fourier transform (in $x$ ) (cf. Heins [9], Levine [15], Miles [16] etc.) or by the method of separation of variables (cf. Rhodes-Robinson [17] where the effect of surface tension of FS is included), thereby obtaining a linear combination of potentials due to the source in an unbounded fluid together with an 'image' potential in the FS boundary condition.

In the present paper we consider a two-fluid medium and dertve velocity potentials due to a vertical line source present in either of the fluids whose strength varies harmonically with time and also with the co-ordinate measured along its length. This is the same as deriving the source potentials in a two-fluid medium for the reduced two dimensional Helmholtz's equation. The corresponding problem for the two-dimensional Laplace's equation was considered in [4]. When the strength of the line source is made independent of the co-ordinate along its length, known results for a two-fluid medium are recovered. When the density of the upper fluid is made zero, the results derived here reduce to corresponding known results for an on-fluid medium.

2. STATEMENT OF THE PROBLEM.

We consider a two-fluid medium, both the fluids being incompressible and inviscid. The mean $S S$ is horizontal and taken as the $x z$-plane $y$-axis pointing vertically downwards. A line source is assumed to be present in either of the fluids and the $y$-axis is chosen to pass through the singular point so that the point of singularity is situated efther at $(0, n)$ or $(0,-n)(n>0)$. The strength of the line source is assumed to vary sinusoidally with time as well as with $z$. Let $\rho_{1}, \rho_{2}$ be the densities of the lower and upper fluids respectively so that $\rho_{1}>\rho_{2}$. The motion is assumed to be irrotational and is of small amplitude, and can be described, by velocity potentials $\operatorname{Re}\left\{p_{j}(x, y, z) \exp (-i \omega t\}(j=1,2)\right.$, where $\omega$ is the circular frequency. $D$ 's satisfy the three-dimensional Laplace's equation in respective fluid regions except at the point of singularity where it exists. The linearized SS conditions are

$$
\begin{array}{ll}
K s_{1}+\frac{\partial z_{1}}{\partial y}=s\left(K P_{2}+\frac{\partial p_{2}}{\partial y}\right), y=0, \\
\frac{\partial p_{1}}{\partial y}=\frac{\partial p_{2}}{\partial y} \quad \text { on } & y=0,
\end{array}
$$

where $k=\omega^{2} / g, g$ being the gravity and $s=\mu_{2} / \mu_{1}<1$. If the lower fluid is of depth ' $h$ ' below the mean SS, then 


$$
\frac{\partial p_{1}}{\partial y}=0 \quad \text { on } \quad y=h \text {, }
$$

otherwise,

$$
\left|\operatorname{grad} p_{1}\right|+0 \text { as } y+-\infty
$$

Also $\left|\operatorname{grad} \otimes_{2}\right|+0$ as $y+-\infty$.

Further, $P_{1}, P_{2}$ satisfy the radiation condition that both represent outgoing waves in the far fiels as $|x| \rightarrow \infty$.

Assuming the z-variation of the strength of the line source as $\exp (i v z)$, it is possible to extract the z-variation completely from the functions $\triangleright_{j}(x, y, z)$. Thus we can write

$$
\mathbb{S}_{j}(x, y, z)=\phi_{j}(x, y) \exp (i \vee z) \quad j=1,2
$$

where now $\phi_{j}$ 's satisfy the two-dimensional modified Helmholtz's equation

$$
\left(\nabla^{2}-\nu^{2}\right) \phi_{j}=0 \text { in } D_{j}
$$

except at a point of singularity, where $D_{1}, D_{2}$ denote respectively the regions occupled by lower and upper fluids and $\nabla^{2}$ is the two-dimensional Laplacian operator. Near a point of singularity the potential behave as $K_{0}(U R)$ which is a typical singular solution of Helmholtz's equation, $K_{0}(z)$ being the modified Bessel function of second kind and $R$ being the distance from the point. The boundary conditions are

$$
\begin{array}{rlrl}
K \phi_{1}+\frac{\partial \phi_{1}}{\partial y} & =s\left(K \phi_{2}+\frac{\partial \phi_{2}}{\partial y}\right), & y=0 ; \\
\frac{\partial \phi_{1}}{\partial y} & =\frac{\partial \phi_{2}}{\partial y}, & y & =0 ; \\
\frac{\partial \phi_{1}}{\partial y} & =0, & y & =h
\end{array}
$$

when the lower fluid is of finite depth, otherwise,

$$
\left|\nabla_{1}\right|+0 \text { as } \mathrm{y} \rightarrow \infty
$$

when the lower fluid is of infinite depth; also

$$
\left|\nabla_{\phi_{2}}\right|+0 \text { as } \mathrm{y}+-\infty
$$

and finally, $\phi_{1}, \phi_{2}$ satisfy the radiation condition in the far field as

$$
|\mathbf{x}|+\infty \text {. }
$$

Thus $\phi_{1}, \phi_{2}$ satisfy a boundary value problem (BVP) described by (2.1) to (2.6). In section 3 we will decompose this BVP into two BVP's by defining two sets of component potentials where the first set accounts for the singularity in the medium but die out in the far field while the second set is non-singular but accounts for the radiation condition in the far field as $|x|+\infty$. In sections 4 and 5 we will obtain solutions to these BVP's assuming the lower fluid to be of infinite and finite depth respectively, thereby deriving the source potentials in the two fluids completely. 
3. DECOMPOSITION INTO TWO BOUNDARY VALUE PROBLEMS.

We define potentials $\psi_{j}(x, y), x_{j}(x, y)(j=1.2)$ such that

$$
\phi_{j}=\dot{\psi}_{j}+x_{j} j=1,2
$$

where $\dot{\psi}_{j}$ satisfy

$$
\left(\nabla^{2}-\nu^{2}\right) \psi_{j}=0
$$

in $D_{j}$ except at a point of singularity, and near a singularity the appropriate conditions are

$$
\begin{array}{ll}
\psi_{j}+K_{0}(\nu R) \text { as } & R+0 . \\
\psi_{1}=s \psi_{2}, & y=0, \\
\frac{\partial \psi_{1}}{\partial y}=\frac{\partial \psi_{2}}{\partial y}, & y=0, \\
\psi_{1}, \psi_{2}+0 \text { as } & \left(x^{2}+y^{2}\right)^{1 / 2} \rightarrow \infty
\end{array}
$$

In $D_{1}, D_{2}$ respectively. Thus $\dot{\psi}_{1}, \psi_{2}$ satisfy the BVP described by (3.2) to (3.6) (hereinafter $P 1$ ). Then $X_{1}, X_{2}$ satisfy the BVP (hereinafter $P 2$ ) described by

$$
\begin{aligned}
& \left(\nabla^{2}-v^{2}\right) x_{j}=0 \quad \text { in } \quad D_{j}(j=1,2), \\
& k x_{1}+\frac{\partial}{\partial y}\left(\psi_{1}+x_{1}\right)=s\left\{k x_{2}+\frac{\partial}{\partial y}\left(\psi_{2}+x_{2}\right)\right\}, y=0 ; \\
& \frac{\partial x_{1}}{\partial y}=\frac{\partial x_{2}}{\partial y}, y=0 \\
& \frac{\partial x_{1}}{\partial y}=-\frac{\partial \psi_{1}}{\partial y}, \quad y=h
\end{aligned}
$$

if there is a bottom to the lower fluid, otherwise,

$$
\begin{aligned}
& \left|\nabla_{x_{1}}\right|+0 \text { as } y \rightarrow \infty, \\
& \left|\nabla_{x_{2}}\right|+0 \text { as } y+-\infty,
\end{aligned}
$$

and finally, $x_{1}, x_{2}$ satisfy the radiation condition in the $f$ ar field as

$$
|\mathbf{x}|+\infty
$$

In the conditions (3.8) and (3.10a), $\psi_{1}$ and $\psi_{2}$ are assumed to be known (solution of P1).

4. LOWER FLUID OF INFINITE DEPTH.

(i) Wave Source in the Lower Fluid. In this case we seek a solution to the BVP described by $(2.1)$ to $(2.6)$ where $\phi_{1}+K_{0}(\nu r)$ as $r=\left\{x^{2}+(y-n)^{2}\right\}^{1 / 2} \rightarrow 0$. Thus in Pl the precise form of $(3.2)$ and the condition (3.3) are

$$
\begin{aligned}
& \left(\nabla^{2}-\nu^{2}\right) \psi_{1}=0, y>0 \quad \text { except at }(0, n), \\
& \left(\nabla^{2}-\nu^{2}\right) \psi_{2}=0, y<0, \psi_{1}+K_{0}(\nu r) \text { as } r+0
\end{aligned}
$$


Let

$$
\psi_{1}=K_{0}(\nu r)+c_{1} K_{0}\left(\nu r^{*}\right) \psi_{2}=c_{2} K_{0}(\nu r)
$$

where $\mathrm{r}^{*}=\left\{\mathrm{x}^{2}+\left(\mathrm{y}+n^{2}\right)\right\}^{1 / 2}$ is the distance from the image point and $c_{1}, c_{2}$ are unknown constants. Clearly $\psi_{1}, \dot{\psi}_{2}$ as given above satisy the equations (4.1) and the conditions (4.2) and (3.6). We choose $c_{1}$ and $c_{2}$ such that the conditions (3.4) and (3.5) are satisfied.

The following integral representations will be needed in our calculation

$$
\begin{array}{ccc}
\frac{\partial}{\partial y} k_{0}(\nu r)=\mp \int_{\nu}^{\infty} k \zeta^{-1} \cos \zeta x \exp \{\mp k(y-n)\} d k, & y \geqslant n, \\
\frac{\partial}{\partial y} k_{0}\left(\nu r^{*}\right)=\mp \int_{\nu}^{\infty} k \zeta^{-1} \cos \zeta x \exp \{\mp k(y-n)\} d k, & y \geqslant-n, \\
\text { where } \zeta=\left(k^{2}-v^{2}\right)^{1 / 2} \text { and the upper (1ower) sign is for } & y>(<) n .
\end{array}
$$

Thus

$$
\begin{aligned}
& \left.\frac{\partial}{\partial y} \begin{array}{c}
K_{0}(\nu r) \\
K_{0}\left(\nu r^{*}\right)
\end{array}\right|_{y=0}=\mp \int_{\nu}^{\infty} k \zeta^{-1} \cos \zeta x \exp (-k n) d k, \\
& \left.\frac{\partial}{\partial y} K_{K_{0}(\nu r)}\left(\nu r^{*}\right)\right|_{y=h} ^{\infty}=-\int_{\nu} k \zeta^{-1} \cos \zeta x \exp \{-k(h \overline{+} n)\} d k .
\end{aligned}
$$

Conditions (3.4) and (3.5) give after making use of appropriate integral representations given above

$$
1+c_{1}=s c_{2}, 1-c_{1}=c_{2},
$$

from which we obtain

$$
c_{1}=(1-s)(1+s)^{-1}, c_{2}=2(1+s)^{-1} \text {. }
$$

Hence

$$
\begin{aligned}
& \psi_{1}=\mathrm{K}_{0}(\nu \mathrm{r})-\frac{1-\mathrm{s}}{1+\mathrm{s}} \mathrm{K}_{0}\left(\nu \mathrm{r}^{\star}\right) \\
& \psi_{2}=\frac{2}{1+\mathrm{s}} \mathrm{K}_{0}(\nu \mathrm{r})
\end{aligned}
$$

Again, let

$$
\begin{aligned}
& x_{1}=\int_{\nu}^{\infty} A \zeta^{-1} \cos \zeta x \exp (-k y) d k, y>0, \\
& x_{2}=\int_{\nu}^{\infty} B \zeta^{-1} \cos \zeta x \exp (k y) d k, y<0,
\end{aligned}
$$

where A,B are unknown functions of $k$. Clearly $x_{1}, x_{2}$ given above satisfy (3.7), $(3.10 \mathrm{~b})$ and $(3.11)$. The contour in the integrals is to be chosen in such a way that the radiation condition is satisfied automatically. This will be shown in the sequel. The conditions (3.8) and (3.9) lead to

$$
\begin{aligned}
(K-k) A-s(K+k) B & =2(1-s)(1+s)^{-1} \exp (-k 1), \\
A+B & =0 .
\end{aligned}
$$


Thus

$$
A, B= \pm 2(1+s)^{-1} k(k-M)^{-1} \exp (-k n)
$$

where

$$
M=(1+s)(1-s)^{-1} \mathrm{~K} .
$$

Hence

$$
\begin{aligned}
& p_{1}=K_{0}(\nu r)-\frac{1-s}{1+s} K_{0}\left(\nu r^{*}\right)+\frac{2}{1+s} \int_{\nu}^{\infty} \frac{k}{(k-M) \zeta} \cos \zeta x \exp \{-k(y+n)\} d k \\
& p_{2}=\frac{2}{1+s} K_{0}(v r)-\frac{2}{1+s} \int_{\nu}^{\infty} \frac{k}{(k-M) \zeta} \cos \zeta x \exp (k(y-n)) d k
\end{aligned}
$$

where the contour is indented below the pole at $k=M$ to ensure the radiation condition at infinity. To establish this, we replace $2 \cos \zeta x$ in the integrals by exp (i $\zeta|x|)+\exp (-i \zeta|x|)$. The contour in the integral involving exp (i $\zeta|x|)$ is deformed into a line from $\nu$ to $X$ (where $X$ is a large positive number) on the real axis with an indentation below the pole at $k=M$, the quarter of a circle of radius $X$ in the first quadrant, the imaginary axis from $i x$ to 0 and a line from 0 to $v$ just above the cut from $k=-\nu$ to $\nu$ in the complex $k$-plane. It is being assumed that $\nu<M$. (In fact if we assume an incident wave field represented by $\phi_{1}^{\text {inc }}=-\exp$ $\{-M y+i(M \cos \alpha x+M \sin \alpha z)\}, y<0$ then $\nu=M \sin \alpha$. However see section 6). In this case there will be a contribution from the pole at $k=M$. As $X+\infty$, the contribution from the circular arc will be exponentially small. Similarly in the integral involving $\exp (-i \zeta|x|)$ the contour is deformed into a line from 0 to $v$ below the cut, a line from $\nu$ to $X$ on the real line with an indentation below the point $k=M$, the quarter of a circle of radius $X$ in the fourth quadrant, and the imaginary axis from $-i x$ to 0 . In this case as the point $k=M$ lies outside the closed contour, there will be no contribution to the integral from this. As $\mathrm{X}+\infty$, the contribution from the circular arc will be exponentially small. The contribution from the real line from 0 to $\nu$ above and below the cut from $k=-v$ to $v$ will cancel out. Comb-ining the two integrals we will finally obtain the alternative representations (which account for the radiation condition in the far fleld as $|x|+\infty$ for $\phi_{1}, \phi_{2}$ as

$$
\begin{aligned}
p_{l} & =K_{0}(\nu r)-\frac{1-s}{1+s} K_{0}\left(\nu r^{*}\right) \\
& +\frac{2}{1+s}\left[\pi i M N^{-1} \exp \{-M(y+n)+i N|x|\}\right. \\
& \left.+\int_{0}^{\infty} \frac{k\{k \cos k(y+n)-M \sin k(y+n)\} \exp \left\{-\left(k^{2}+v^{2}\right)^{1 / 2}|x|\right\}}{\left(k^{2}+M^{2}\right)\left(k^{2}+v^{2}\right)^{1 / 2}} d k\right]
\end{aligned}
$$

and

$$
\begin{gathered}
\phi_{2}=-\frac{2}{1+s}\left[n i M N^{-1} \exp \{M(y-n)+i N|x|\}\right. \\
\left.+\int_{0}^{\infty} \frac{\left.k\{k \cos k(y-\eta)-M \sin k(y-n)\} \exp \left\{-k^{2}+\eta^{2}\right)^{1 / 2}|x|\right\}}{\left.k^{2}+M^{2}\right)\left(k^{2}+v^{2}\right)^{1 / 2}} d k\right]
\end{gathered}
$$

where $\quad N=\left(M^{2}-\nu^{2}\right)^{1 / 2}$. 
(ii) Wave Source in the Upper Fluid. In this case

$$
\varphi_{2}+K_{0}\left(\nu r^{*}\right) \text { as } r^{*}+0 \text { so that } \psi_{2}+K_{0}\left(\nu r^{*}\right) \text { as } r^{*}+0 \text {. }
$$

By writing

$$
\dot{\psi}_{1}=c_{1} K_{0}\left(\nu r^{*}\right), \quad \psi_{2}=c_{2} K_{0}(\nu r)+K_{0}\left(\nu r^{*}\right)
$$

and $x_{1}, x_{2}$ the same integrals as in (4.4) (with different $A$ and B) we will similarly obtain

$$
\begin{aligned}
& \phi_{1}=\frac{2 s}{1+s}\left[K_{0}\left(\nu r^{*}\right)-\psi_{\nu}^{\infty} \frac{k}{(k-M) \zeta} \cos \zeta x \exp \{-k(y+n)\} d k\right] \\
& \phi_{2}=K_{0}\left(\nu r^{*}\right)+\frac{1-s}{1+s} K_{0}(\nu r)+\frac{2 s}{1+s} \psi_{\nu}^{\infty} \frac{k}{(k-M) \zeta} \cos \zeta x \exp \{k(y-n)\} d k
\end{aligned}
$$

where the contour is indented below the pole at $k=M$ to ensure the radiation condition at infinity. Alternative representation for $\phi_{1}, \phi_{2}$ can be obtained following the same method mentioned above as

$$
\begin{aligned}
& \phi_{1}=\frac{2 s}{1+s}\left[K_{0}\left(\nu r^{*}\right)-\text { the terms in the square bracket in }(4.8)\right], \\
& \mu_{2}=K_{0}\left(\nu r^{*}\right)+\frac{1-s}{1+s} K_{0}(\nu r)+\frac{2 s}{1+s}[\text { the terms in the square bracket in }(4.9)] .
\end{aligned}
$$

5. LOWER FLUID OF FINITE DEPTH.

(i) Wave Source in the Lower Fluid. In this case $\dot{\psi}_{1}, \psi_{2}$ are the same as in Section $4(i)$, while $x_{1}, x_{2}$ satisfy P2 with the condition (3.10a) in place of (3.10b). Let

$$
\begin{aligned}
& x_{1}=\int_{\nu}^{\infty} \zeta^{-1} \cos \zeta x\{A \cosh k(h-y)+B \sinh k y\} d k, 0<y<h, \\
& x_{2}=\int_{\nu}^{\infty} c \zeta^{-1} \cos \zeta x \exp (k y) d k, y<0 .
\end{aligned}
$$

$x_{1}, x_{2}$ given above obviously satisfy (3.7) and (3.11). The SS conditions (3.8), (3.9) and the bottom condition (3.10a) yield the following three equations for the derivation of $\mathrm{A}, \mathrm{B}, \mathrm{C}$.

$$
\begin{aligned}
& A(K \cosh k h-k \sinh k h)+k B-s(K+k) C=-2(1-s)(1+s)^{-1} k \exp (-k n), \\
& A \sinh k h-B+C=0 \\
& B \cosh k h=\exp \{-k(h-n)\}-(1-s)(1+s)^{-1} \exp \{k(y+n)\}
\end{aligned}
$$

Solving for $A, B, C$ we obtain

$$
\begin{aligned}
& \phi_{1}=K_{0}(\nu r)-\frac{1-s}{1+s} K_{0}\left(\nu r^{*}\right) \\
&+\frac{2}{1+s} \int_{\nu}^{\infty}\left[\frac{1}{\Delta} \exp (-k h)\{s(K+k)-k\}(s i n h k n+s \cosh K n) \operatorname{sech} k h\right. \\
&-(1-s) \exp (-k \eta) \cosh k(h-y) \\
&+\exp (-k h)(\sinh k n+s \cosh k \eta) \operatorname{sech} k h \sinh k y] \frac{\cos \zeta x}{\zeta} d k
\end{aligned}
$$




$$
\begin{aligned}
\Phi_{2}=\frac{2}{1+s} & K_{0}(v r) \\
& +\frac{2}{1+s} \int_{v}^{\infty}[(\sinh k n+s \cosh k n) \text { sech } k h \exp \{-k(h-y)\} \\
& -\frac{1}{\Delta}\{\exp (-k h)\{s(k+k)-k\}(\sinh k n+s \cosh k n) \operatorname{sech} k h \\
& -(1-s) k \exp (-k n)\} \sinh k h \exp (k y)] \frac{\cos \zeta x}{\zeta} d k,
\end{aligned}
$$

where $\Delta(k)=K \cosh k h+\{s(K+k)-k\}$ sinh $k h$,

and the contour in the integrals is indented below the pole at $k=k_{0}$ which is the only real positive zero of $\Delta(k)$, to ensure the satisfaction of the radiation condtion at infinity.

Note that poles do not occur at $k h= \pm \frac{\pi i}{2}, \pm \frac{3}{2} \pi 1, \ldots$. The only poles occur at the zeros of $\Delta(k) . \Delta(k)$ has two real zeros, one is positive, $k_{0}$ say, and the other is negative. When $s=0$, magnitudes of these real zeros become the same. The remaining zeros of $\Delta(k)$ are complex in general. When $s=0$, the complex zeros become purely imaginary (see Rhodes-Robinson [18] with surface tension put equal to zero). To ensure the radiation condition in the far field as $|x| \rightarrow \infty$, the same steps of section 4 (i) can be followed in the deformation of the contours in the first and fourth quadrants with the modification that the contours are indented below the pole at $k=k_{0}$, and the large radius of the circular arc in the first and fourth quadrants is chosen in such a way that no complex zero of $\Delta(k)$ is crossed. The far field behaviour will come only from the contribution to the integrals at $k=k_{0}$ (when the contour is deformed in the first quadrant), other contributions from the imaginary axis, from the poles at complex zeros lying in the first and fourth quadrants will die out in the far field as $|x| \rightarrow \infty$. The contribution from the real line from 0 to $v$ above and below the cut from $k=-\nu$ to $\nu$ will cancel out. Thus as $|x|+\infty$

$$
\begin{aligned}
& \dot{\psi}_{1}+D_{1} \frac{\cosh k_{0}(h-y)}{\sinh k_{0} h} N_{0}^{-1} \exp \left(i N_{0}|x|\right), \\
& \psi_{L} \rightarrow-D_{1} \exp \left(k_{0} h\right) N_{0}^{-1} \exp \left(i N_{0}|x|\right)
\end{aligned}
$$

where $\quad \mathrm{N}_{0}=\left(\mathrm{k}_{0}^{2}-v^{2}\right)^{1 / 2}$,

and

$$
\begin{aligned}
& D_{1}=\frac{2}{1+s} \pi i {\left[\exp \left(-k_{0} h\right)\left\{s\left(k_{0}+K\right)-k_{0}\right\}\left(\sinh k_{0} n+s \cosh k_{0} n\right) \text { sech } k_{0} h\right.} \\
&\left.-(1-s) k_{0} \exp \left(-k_{0} n\right)\right] \frac{\sinh k_{0} h}{\Delta^{\prime}\left(k_{0}\right)}
\end{aligned}
$$

where $\quad \Delta^{\prime}\left(k_{0}\right)=\left.\frac{d}{d k} \Delta(k)\right|_{k=k_{0}}$

(ii) Wave Source Submerged in the Upper Fluid. In this case $\psi_{1}, \psi_{2}$ are the same as given in Section 4 (ii) while $x_{1}, x_{2}$ may be assumed to have the same type of representation given in (5.1) (with different A, B, C). Then A, B, C satisfy 

$A \sinh k h-B+C=0$,
B $\cosh k h=2 s(1+s)^{-1} \exp \{-k(h+n)\}$

Thus we will finally obtain

$$
\begin{aligned}
& \phi_{1}= \frac{2 s}{1+s} K_{0}\left(\nu r^{*}\right)+\frac{2 s}{1+s} \int_{\nu}^{\infty}\left\{\frac{(1-s) k+\{s K-(1+s) k\} \exp (-k h) s e c h k h}{\Delta}\right. \\
&\exp (-k n) \cosh k(h-y)+\exp \{-k(h+n)\} \operatorname{sech} k h \sinh k y\} \frac{\cos \zeta x}{\zeta} d k, \\
& \phi_{2}= K_{0}\left(\nu r^{*}\right)+\frac{1-s}{1+s} K_{0}(\nu r)+\frac{2 s}{1+s} \int_{\nu}^{\infty}[\exp (-k h) \operatorname{sech} k h \\
&\left.-\frac{1}{\Delta}\{(1-s) k+\{s K-(1+s) k\} \exp (-k h) \operatorname{sech} k h\} \quad \sinh k h \exp k(y-n)\right] \frac{\cos \zeta x}{\zeta} d k
\end{aligned}
$$

As $|x|+\infty$,

$$
\begin{aligned}
& \phi_{1} \rightarrow D_{2} \frac{\cosh k_{0}(h-y)}{\sinh k_{0} h} N_{0}^{-1} \exp \left(i N_{0}|x|\right), \\
& \phi_{2} \rightarrow-D_{2} \exp \left(k_{0} y\right) N_{0}^{-1} \exp \left(i N_{0}|x|\right),
\end{aligned}
$$

where

$$
\begin{gathered}
D_{2}=2 s(1+s)^{-1} \pi_{i}\left[(1-s) k_{0}+\left\{s K-(1+s) k_{0}\right\} \exp \left(-k_{0} h\right) \operatorname{sech} k_{0} h\right] \\
\frac{\sinh k_{0} h}{\Delta^{\prime}\left(k_{0}\right)} \exp \left(-k_{0} n\right),
\end{gathered}
$$

and $\mathrm{N}_{0}$ is given by $(5 \cdot 6)$.

6. CONCLUSION.

We have derived in the present paper source potentials for the two-dimensional modified Helmholtz's equation in a two-fluid medium. The parameter $\nu$ in the Helmholtz's equation has been assumed to be less than the wave parameter $M$ (for infinite depth of the lower fluid) or $k_{0}$ (for finite depth of the lower fluid). However, if $v$ is greater than the wave parameter then the potentials will no longer represent outgoing waves in the far field, rather they will die out in the far field (see the corresponding one-fluid case with surface tension in the FS in [16]).

Making $s \rightarrow 0$ in the above results, source potentials in an one-fluld medium ([16] with surface tension put equal to zero) can be recovered. Making $\nu \rightarrow 0$, potentials due to only time-harmonic line source in a two-fluid medium [3] can be recovered. One can also include the effect of surface tension of the sS in these results. 


\section{REFERENCES}

1. THORNE, R.C. Multipole Expansion in the Theory of Surface Waves. Proc. Camb. Phil. Soc. 49 (1953), 701-716.

2. RHODES-ROBINSON, P.F. Fundamental Singularities in the Theory of Water Waves with Surface Tension. Bull. Austral. Math. Soc. 2 (1970) . 317-333.

3. GORGUI, M.A. and KASSEM, S.E. Basic Singularities in the Theory of Internal Waves. Quart. J. Mech. App1. Math. 31 (1978). 31-48.

4. RHODES-ROBINSON, P.F. On Waves at Interface Between Two Liquids. Math. Proc. Camb. Phil. Soc. 88 (1980), 183-191.

5. MANDAL, B.N. Some Basic Singularities in the Theory of Internal Waves in a TwoFluid Medium in the Presence of Surface Tension. J. Tech. 26 (1981), 11-22.

6. CHAKRABARTI, R.N. and MANDAL, B.N. Singularities in a Two-Fluid Medium. Internat. J. Math. and Math. Sci. 6 (1983), 737-754.

7. CHAKRABARTI, R.N. Singularities in a Two-Fluid Medium with Surface Tension at Their Surface of Separation. Bull. Cal. Math. Soc. 75 (1983), 271-282.

8. KASSEM, S.E.Multipole Expansions for Two Superposed Fluids, each of Finite Depth. Math. Proc. Camb. Phil. Soc. 91 (1982), 323-329.

9. HEINS, A.E. Water Waves Over a Channel of Finite Depth With a Submerged Plane Barrier. Canad. J. Math. 2 (1950), 210-222.

10. GREEN, T.H. and HEINS, A.E. Water Waves Over a Channel of Infinite Depth. Quart. Appl. Math. 11 (1953), 201-214.

11. MANDAL, B.N. and GOSWAMI, S.K. A Note on the Diffraction of an Obliquely Incident Surface Wave By a Partially Immersed Fixed Vertical Barrier. Appl. Sc1. Res. 40 (1983), 345-353.

12. MANDAL, B.N. and GOSWAMI, S.K. The Scattering of an Obliquely Incident Surface Wave By a Submerged Fixed Vertical Plate. J. Math. Phys. 25 (1984), 1780-1783.

13. MANDAL, B.N. and GOSWAMI, S.K. A Note . on the Scattering of Surface Waves obliquely Incident on a Submerged Fixed Vertical Barrier. J. Phys. Soc. Japan 53 $(1984), 2980-2987$.

14. MANDAL, B.N. and GOSWAMI, S.K. Scattering of Surface Waves Obliquely Incident on a Fixed Half Immersed Circular Cylinder. Math. Proc. Camb. Phil. Soc. 96 (1984), 359-369.

15. LEVINE, H. Scattering of Surface Waves By a Submerged Circular Cylinder. J. Math. Phys. 6 (1965), 1231-1234.

16. MILES, J.W. Surface-wave Scattering Matrix For a Shelf. J. Fluid. Mech. 23 $(1965), 399-414$.

17. RHODES-ROBINSON, P.F. On Forced Three-dimensional Surface Waves In a Channel In the Presence of Surface Tension. Proc. Camb. Phil. Soc. 75 (1974), 405-426.

18. RHODES-ROBINSON, P.F. On the Forced Surface Waves Due To a Vertical Wave-maker In the Presence of Surface Tension. Proc. Camb. Phil. Soc. 70 (1971), 323-337. 


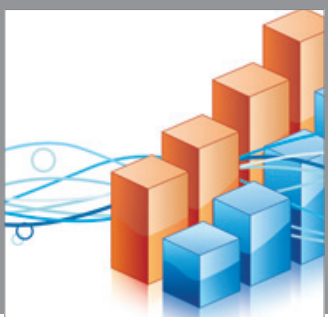

Advances in

Operations Research

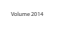

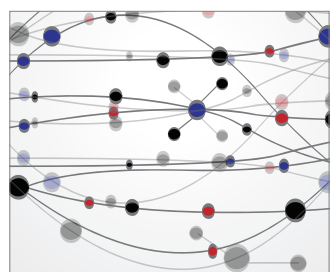

\section{The Scientific} World Journal
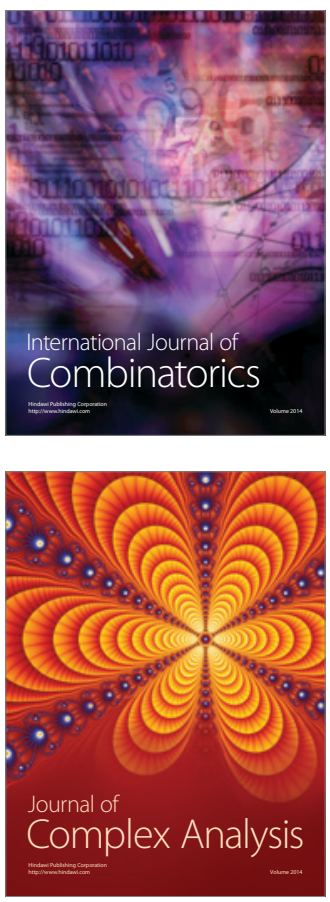

International Journal of

Mathematics and

Mathematical

Sciences
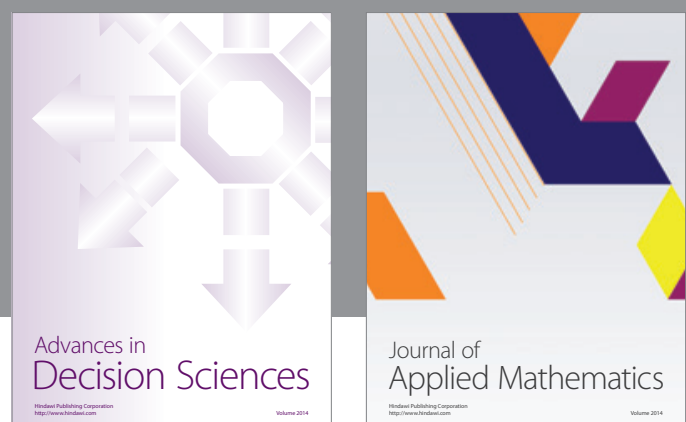

Journal of

Applied Mathematics
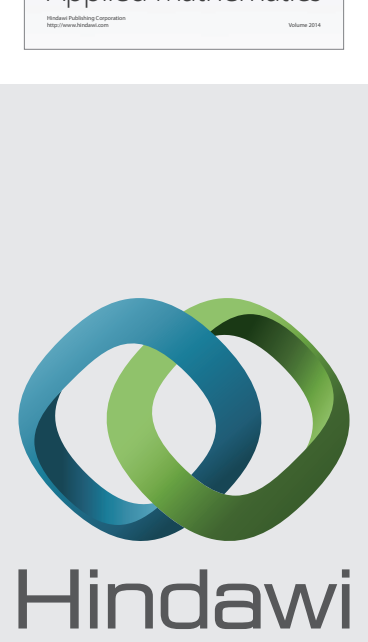

Submit your manuscripts at http://www.hindawi.com
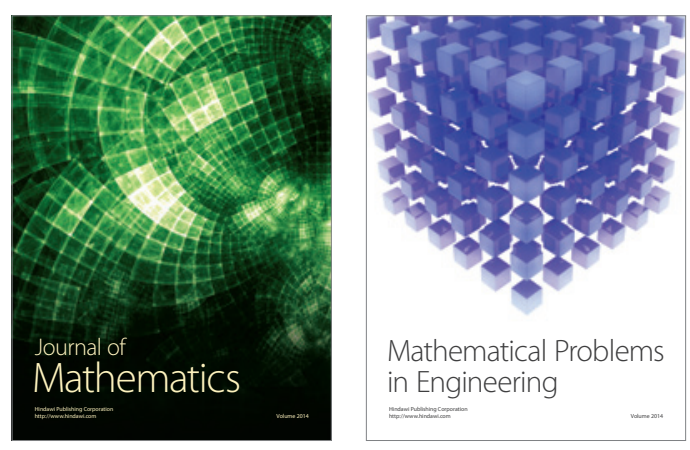

Mathematical Problems in Engineering
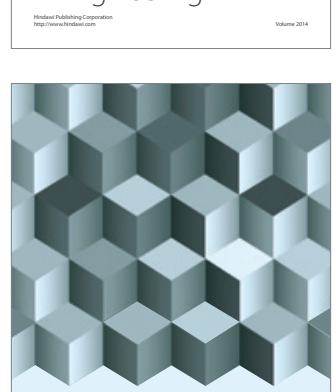

Journal of

Function Spaces
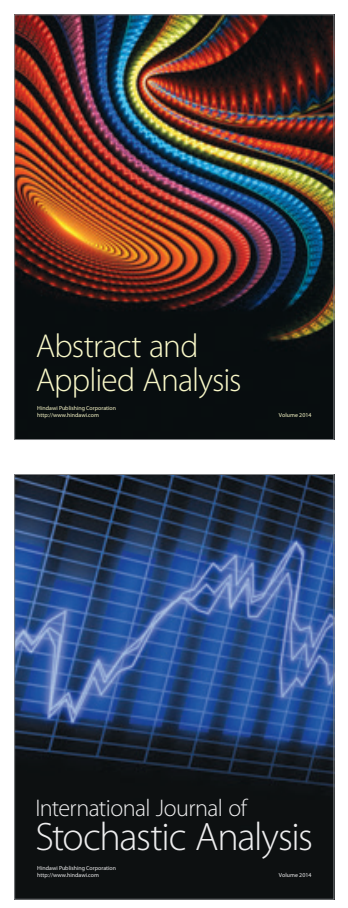

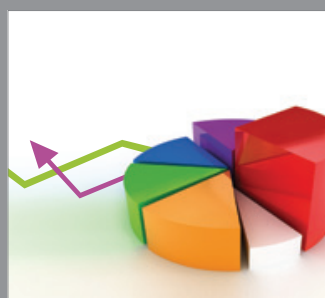

ournal of

Probability and Statistics

Promensencen
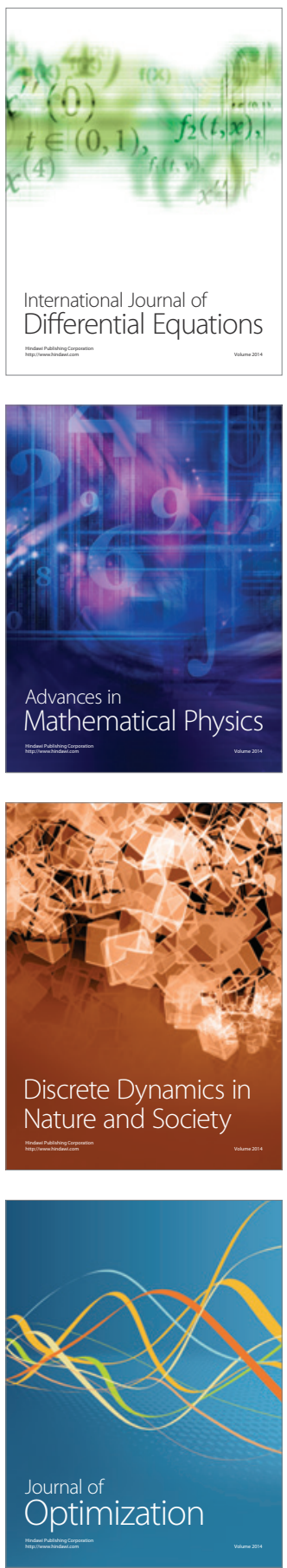